\title{
Specialized adaptation of a lactic acid bacterium to the milk environment: the comparative genomics of Streptococcus thermophilus LMD-9
}

\author{
Yong Jun Goh', Caitlin Goin², Sarah O'Flaherty ${ }^{1}$, Eric Altermann ${ }^{3,4}$, Robert Hutkins ${ }^{{ }^{*}}$ \\ From 10th Symposium on Lactic Acid Bacterium \\ Egmond aan Zee, the Netherlands. 28 August - 1 September 2011
}

\begin{abstract}
Background: Streptococcus thermophilus represents the only species among the streptococci that has "Generally Regarded As Safe" status and that plays an economically important role in the fermentation of yogurt and cheeses. We conducted comparative genome analysis of S. thermophilus LMD-9 to identify unique gene features as well as features that contribute to its adaptation to the dairy environment. In addition, we investigated the transcriptome response of LMD-9 during growth in milk in the presence of Lactobacillus delbrueckii ssp. bulgaricus, a companion culture in yogurt fermentation, and during lytic bacteriophage infection.
\end{abstract}

Results: The S. thermophilus LMD-9 genome is comprised of a $1.8 \mathrm{Mbp}$ circular chromosome $(39.1 \% \mathrm{GC} ; 1,834$ predicted open reading frames) and two small cryptic plasmids. Genome comparison with the previously sequenced LMG 18311 and CNRZ1066 strains revealed 114 kb of LMD-9 specific chromosomal region, including genes that encode for histidine biosynthetic pathway, a cell surface proteinase, various host defense mechanisms and a phage remnant. Interestingly, also unique to LMD-9 are genes encoding for a putative mucus-binding protein, a peptide transporter, and exopolysaccharide biosynthetic proteins that have close orthologs in human intestinal microorganisms. LMD-9 harbors a large number of pseudogenes (13\% of ORFeome), indicating that like LMG 18311 and CNRZ1066, LMD-9 has also undergone major reductive evolution, with the loss of carbohydrate metabolic genes and virulence genes found in their streptococcal counterparts. Functional genome distribution analysis of ORFeomes among streptococci showed that all three $S$. thermophilus strains formed a distinct functional cluster, further establishing their specialized adaptation to the nutrient-rich milk niche. An upregulation of CRISPR1 expression in LMD-9 during lytic bacteriophage DT1 infection suggests its protective role against phage invasion. When co-cultured with L. bulgaricus, LMD-9 overexpressed genes involved in amino acid transport and metabolism as well as DNA replication.

Conclusions: The genome of S. thermophilus LMD-9 is shaped by its domestication in the dairy environment, with gene features that conferred rapid growth in milk, stress response mechanisms and host defense systems that are relevant to its industrial applications. The presence of a unique exopolysaccharide gene cluster and cell surface protein orthologs commonly associated with probiotic functionality revealed potential probiotic applications of LMD-9.

\footnotetext{
* Correspondence: rhutkins1@unl.edu

${ }^{5}$ Department of Food Science and Technology, University of Nebraska-

Lincoln, Lincoln, Nebraska 68583, USA

Full list of author information is available at the end of the article
} 


\section{Background}

Originally described by Orla-Jensen in 1919 [1], Streptococcus thermophilus is a low G $+\mathrm{C}$, Gram-positive, nonmotile, non-spore-forming, catalase-negative, facultative anaerobic, homofermentative lactic acid bacterium that has restricted natural habitats in the bovine mammary mucosa and raw milk. Among the ninety-three currently classified species from the genus Streptococcus [2], S. thermophilus represents the only species that is "Generally Regarded As Safe" (GRAS). Accordingly, S. thermophilus plays a prominent role in food biopreservation. Notably, the species is used extensively in yogurt, cheese, and other dairy fermentations, where it is traditionally paired with Lactobacillus delbrueckii subsp. bulgaricus or Lactobacillus helveticus. In these products, S. thermophilus is responsible for rapid acidification as well as formation of the expected flavor and texture properties [3]. In addition, yogurt cultures have also been linked to various probiotic effects, including the alleviation of lactose intolerance [4], modulation of intestinal microbiota [5], immunostimulation of host production of pro-inflammatory and antiinflammatory cytokines [6,7], and inhibition of specific periodontal pathogens [8].

There are currently 48 completed genome sequences from the genus Streptococcus that are listed in the National Center for Biotechnology Information (NCBI) Microbial Genomes database. In addition, 311 streptococcal genome sequencing projects are currently in progress. Among these, three complete genomes belong to $S$. thermophilus, with a fourth that is in progress. The genome blueprints of S. thermophilus LMD-9 and two other S. thermophilus strains, CNRZ1066 and LMG 18311 [9], have led to the identification of several important features. Not surprisingly, genes associated with virulence in other streptococci are either non-functional or completely absent in S. thermophilus $[9,10]$, suggesting that reductive evolution had occurred during its domestication in the nutritionally-rich milk niche. In addition, the LMD-9 genome contains regions unique to this strain, including genes encoding for the production and regulation of a broad spectrum bacteriocin, Thermophilin 9, genes involved in quorum sensing and competence development [11-16], and several of the clustered regularly interspaced short palindromic repeats (CRISPR) regions not present in both CNRZ1066 and LMG 18311 that are involved in bacteriophage defense $[17,18]$.

Interestingly, long before even the notion of a genome had even been conceived, S. thermophilus was reportedly known "more by the things which it cannot do than by its positive actions" [19]. Numerous studies have led to the realization that this view is no longer accurate and that $S$. thermophilus is indeed genetically equipped to perform numerous biological functions [20]. In this current report, we describe the many unique properties present in this organism, as revealed by comparative genomic analyses of LMD-9 with other S. thermophilus strains as well as commensal and pathogenic streptococcal species. We also present results obtained from transcriptome analysis and in-depth mining of the annotated LMD-9 genome to uncover other genetic features that are potentially relevant to its adaptation and performance in the dairy environment.

\section{Methods}

\section{Genome sequencing}

The genome sequencing of Streptococcus thermophilus LMD-9 (ATCC BAA-491) was described previously [10]. The complete genome sequence and curated annotation of S. thermophilus LMD-9 can be accessed at GenBank under accession number CP000419 (chromosome), CP000420 (cryptic plasmid pSTER_A) and CP000421 (cryptic plasmid pSTER_B).

\section{Bioinformatic analyses}

The GAMOLA [21]/Artemis [22] software suite was used to manage the genome annotation. Protein-encoding open reading frames (ORFs) were identified using the ORF-prediction program Glimmer2 [23]. ORFeome previously predicted by Makarova et al. [10] was used as a reference. A manual inspection was performed in order to verify or, if necessary, redefine the start and stop positions of each ORF based on sequence alignment with proteins in the non-redundant protein database provided by NCBI and potential ribosomal binding sites. Assignment of protein function to ORFs was performed manually using results from several sources. BLASTP [24] was use on both a non-redundant protein database provided by NCBI and clusters of orthologous groups (COG) [25] databases. HMMER [26] was used to identify protein motifs to the PFAM libraries [27]. TMHMM [28] was used to predict transmembrane sequences, and SignalP [29] was used for the prediction of signal peptides. The TatP prediction tool was used to search for proteins that contain a TAT signal peptide and TAT motif [30]. Chromosomal and plasmid maps were constructed using the Microbial Genome Viewer [31] and Clone Manager Professional software (Sci-Ed Software, Cary, NC), respectively.

Whole genome sequences were aligned using the GenomeComp software tool [32] with the external MEGABLAST nucleotide sequence alignment search program available from NCBI (ftp://ftp.ncbi.nih.gov/blast/) and default run parameters. Metabolic pathway mapping of S. thermophilus LMD-9 ORFeome was executed using the software suite PathwayVoyager [33] with the Kyoto 
Encyclopedia of Genes and Genomes (KEGG) online database (http://www.genome.jp/kegg/kegg2.html).

A Functional Genome Distribution analysis (FGD, E. Altermann, in preparation) was performed using 39 publicly available Streptococcus genome sequences, including S. thermophilus LMD-9 (Additional file 1). FGD analyzes the functional similarity between microbes based on their predicted ORFeomes. FGD is a comparative genomics approach to genome-genome comparisons, emphasizing functional relationships rather than ancestral lineages. Briefly, pooled ORFeomes are subjected to all-versus-all analyses, evaluating the level and quality of amino-acid similarities between individual ORFs pairings. Results for each genome-genome combination are then combined into a symmetrical distance matrix and was visualized using the Unweighted Pair Group Method with Arithmetic mean (UPGMA) method [34]. Strain and cluster conserved and specific gene sets were mined based on respective BLAST e-values, using custom developed software. The distance matrix was imported into MEGA4 [35] for visualization. Based on the FGD calculations, ORFeome synteny on amino acid level between all genomes was assessed using ACT [36].

\section{Transcriptome analysis. (i) Bacteriophage infection}

S. thermophilus LMD-9 was incubated in M17 + glucose at $42^{\circ} \mathrm{C}$ until reaching OD625 0.4, then the culture was split. The lytic bacteriophage DT1 was added at a M.O.I. of 1 to one culture as the expression treatment and samples were taken at time points of 5 min before infection and $0,5,10,20,30$, and $40 \mathrm{~min}$ after infection. The cells of the other half of the culture were immediately harvested by centrifugation as the control.

\section{(ii) S. thermophilus and L. bulgaricus synergism in milk}

L. bulgaricus BAA-365 was grown in skim milk for $7 \mathrm{hrs}$ at $42^{\circ} \mathrm{C}$, and then added at an equal volume to $S$. thermophilus LMD-9, also in skim milk. Sterile skim milk at a similar pH was added to a parallel culture of LMD-9 as a control. After 4 hours or when pH 5.5 was reached, cells were collected for RNA isolation.

(iii) Microarray fabrication, hybridization and data analysis Microarrays were fabricated as 60mer oligo-chip arrays generated from the $S$. thermophilus LMD-9 genome (Invitrogen, Carlsbad, CA). Each oligomer was contact-printed using the OminGrid robotic arrayer (GeneMachine, San Carlos, CA), in triplicate, for a total of 4,866 features per microarray. Slides were pre-treated according to the manufacturer's recommendations using a UV cross-linking method to anchor the oligos to the surface of the epoxy slide.

Cells grown under various growth conditions were harvested by centrifugation, and RNAprotect (Qiagen
Inc., Valencia, CA) was used to stop gene expression and stabilize the RNA [37]. RNA isolation was achieved using the chaotropic agent TRI reagent (Molecular Research Center, Inc., Cincinnati, $\mathrm{OH}$ ) according to the manufacturer's instructions, followed by bead-beating and chloroform extraction, purification, and DNase treatment (Turbo DNase, Ambion/Applied Biosystems, Austin, TX). The cDNA was synthesized using Superscript II Reverse Transcriptase (Invitrogen) from $30 \mu \mathrm{g}$ of extracted RNA and directly labeled with two different fluorochromes (Perkin Elmer Inc., Waltham, MA); Cy3 was used to label the treatment group and Cy5 was used to label the control group. The labeled probes were hybridized to the microarray slides in a HybChamber (GeneMachine) for 16 to 20 hours at $43{ }^{\circ} \mathrm{C}$, then the slides were washed in a series of three washing buffers [(1) 1X SSC, 0.03\% SDS, (2) 0.2x SSC, and (3) 0.05 $\mathrm{x}$ SSC] and scanned using a GenePix 4000B scanner (Axon Instruments/Molecular Devices, Inc., Sunnyvale, CA) at $5 \mu \mathrm{m}$ per pixel resolution.

Statistical analysis of the data was based on the median pixel intensities (at wavelengths of 635 and $532 \mathrm{~nm}$ ) generated by the GenePix scanner. The values were normalized between spots and between each of the three replicates performed using LimmaGui software package (http://bioinf.wehi.edu.au/limmaGUI/) with general loess after background correction. The least squares method was used to determine differentially expressed genes, and only those genes with a $\mathrm{p}$ value of $\leq 0.05$ were considered significantly different.

\section{Results and discussion \\ General genome features}

The general genome features of $S$. thermophilus LMD-9 was previously described as part of a multiple lactic acid bacteria genomes sequencing project and comparative analysis [10]. The LMD-9 genome is comprised of a single circular chromosome $(1,856,368 \mathrm{bp} ; 39.1 \% \mathrm{G}+\mathrm{C})$ and two cryptic plasmids, pSTER_A $(4,449 \mathrm{bp} ; 37.0 \% \mathrm{G}+\mathrm{C})$ and pSTER_B (3,361 bp; $35.1 \% \mathrm{G}+\mathrm{C})$ (Figure 1). Collectively, the LMD-9 genome is approximately $60 \mathrm{~kb}$ larger than the genomes of the previously sequenced plasmidfree strains LMG 18311 and CNRZ1066 [9] (Table 1). Despite the modest differences in genome size, all three genomes have the same $G+C$ content of $39.1 \%$. The LMD-9 chromosome contains 1,834 predicted open reading frames (ORFs) that accounts for $81.2 \%$ of the chromosomal sequence. The ORFeome has an overall $\mathrm{G}+\mathrm{C}$ content of $39.93 \%$ and an average gene length of $822 \mathrm{bp}$. Putative biological roles have been assigned for 1,417 (77\%) of the ORFs, whereas the remaining 417 ORFs encode for hypothetical proteins in which no probable function could be predicted. As many as 241 (13\%) ORFs appear to be pseudogenes (Additional file 2). The majority 


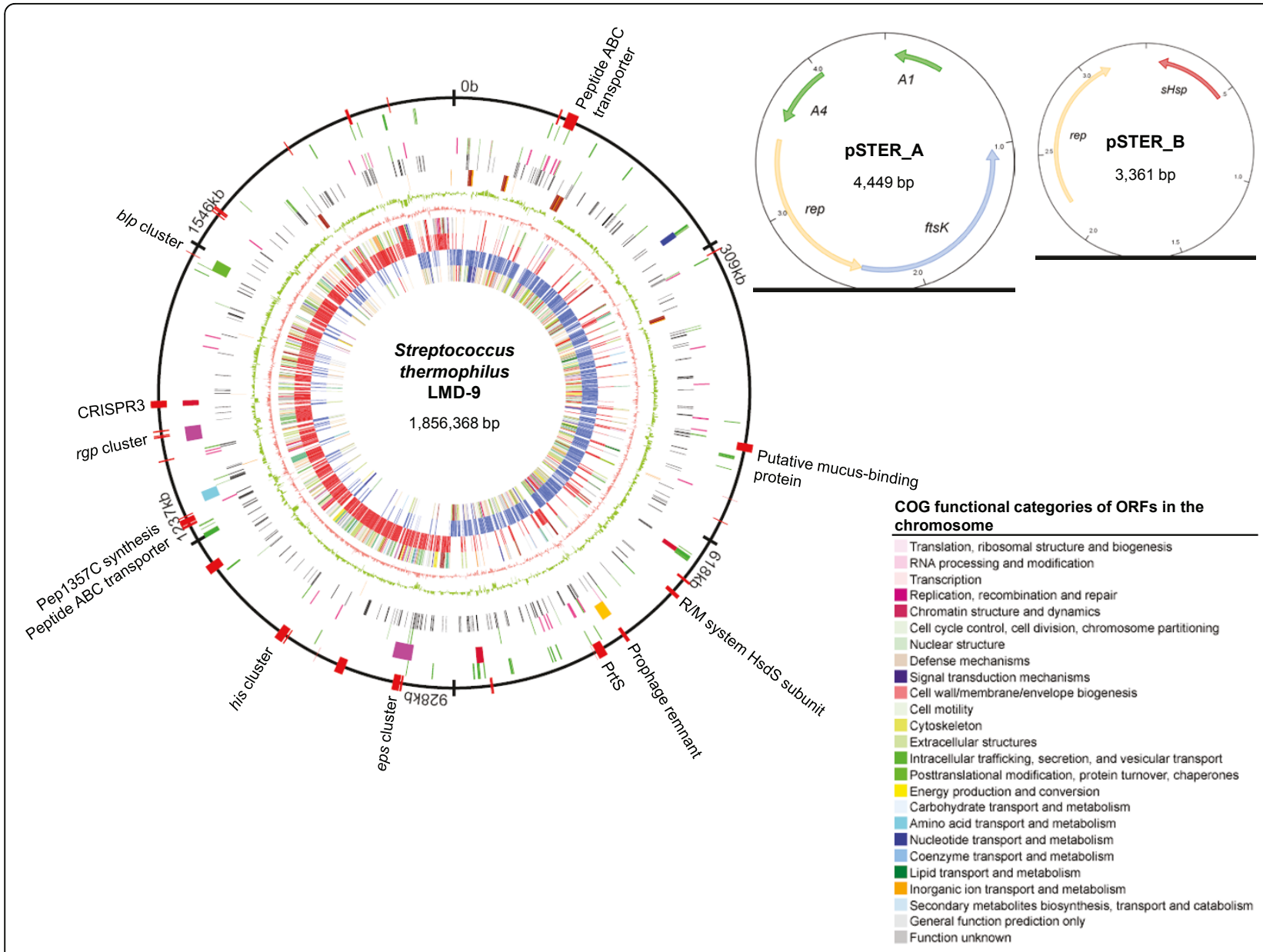

Figure 1 Genome maps of the S. thermophilus LMD-9 chromosome and two cryptic plasmids, pSTER_A and pSTER_B. From the innermost towards the outermost circles of the chromosome map: Circle 1, COG functional classification of ORFs on the forward strand; Circle 2 (blue), ORFs on the forward strand; Circle 3 (red), ORFs on the reverse strand; Circle 4, COG functional classification of ORFs on the reverse strand; Circle 5 (salmon), GC skew; Circle 6 (green), GC composition (\%); Circle 7, rRNAs and tRNAs, shown in brown and orange, respectively; Circle 8 (dark grey), pseudogenes; Circle 9, intact and truncated transposases, shown in red and green, respectively; Circle 10, key features encoded in the chromosome - urease gene cluster (blue), CRISPR regions (dark red), prophage remnant (yellow), eps and rgp clusters (fuchsia), gal-lac cluster (cyan) and blp cluster (green); Circle 11 (green), S. thermophilus-specific genes not present in S. salivarius SK126 draft genome; Circle 12 (red), LMD-9 specific genes or chromosomal regions not present in both CNRZ1066 and LMG 18311. Selected features within the unique regions are indicated. Genes encoding the Pep1357C cyclic peptide was previously identified by Ibrahim et al. [15]. Color designation for COG classification of ORFs in Circle 1 and 4 is shown at the bottom right legend.

of these pseudogenes encode for transposases, hypothetical proteins, carbohydrate transport and metabolism, and transcriptional regulators.

\section{Genome comparison with S. thermophilus LMG 18311 and} CNRZ1066

Approximately $83 \%$ of the $S$. thermophilus LMD-9 ORFeome are common to both CNRZ1066 and LMG 18311 and constitutes the backbone of these three strains. The latter two strains shared about 93 to $94 \%$ of their ORFs [9]. As observed previously [38], the LMD-9 genome exhibited a lesser degree of global synteny when compared to both LMG 18311 and CNRZ1066, although all three genomes are essentially collinear (Figure 2).

\section{(i) LMD-9 specific regions}

Genome alignment revealed chromosomal regions of approximately $137 \mathrm{~kb}(7.4 \%)$ and $144 \mathrm{~kb}(10.6 \%)$ in the LMD-9 strain that are not present in CNRZ1066 and LMG 18311, respectively. These genomic segments correspond mainly to 73 and 65 regions of $>50$ bp specific in LMD-9 when compared to CNRZ1066 and LMG 18311 genomes, respectively. Collectively, an approximate $114 \mathrm{~kb}$ of LMD-9 chromosomal regions encompassing 127 ORFs (representing 6.9\% of the LMD-9 ORFeome) 
Table 1 General genome features of S. thermophilus

\begin{tabular}{|c|c|c|c|}
\hline \multirow[b]{2}{*}{ Feature } & \multicolumn{3}{|c|}{ S. thermophilus strain } \\
\hline & LMD-9 & CNRZ1066 & LMG 18311 \\
\hline & Danisco & Yogurt isolate (France) & Yogurt isolate (UK) \\
\hline Origin of strain & (USA) & (France) & $(\mathrm{UK})$ \\
\hline Size of chromosome (bp) & $1,856,368$ & $1,796,226$ & $1,796,846$ \\
\hline $\mathrm{G}+\mathrm{C}$ content $(\%)$ & 39.08 & 39.08 & 39.09 \\
\hline No. of predicted ORFs & 1,834 & 1,915 & 1,890 \\
\hline ORFs with putative functions & $1,417(77 \%)$ & $1,372(71.6 \%)$ & $1,376(72.8 \%)$ \\
\hline Coding density & $81 \%$ & $84 \%$ & $84 \%$ \\
\hline Pseudogenes (\% ORFs) & $241(13.1 \%)$ & $182(9.5 \%)$ & $180(9.5 \%)$ \\
\hline No. of rRNA operons & 6 & 6 & 6 \\
\hline No. of tRNAs & 67 & 67 & 67 \\
\hline Prophage remnant & 1 & 1 & 0 \\
\hline Plasmid & 2 & 0 & 0 \\
\hline Genbank Accession no. & СР000419 & СР000024 & СР000023 \\
\hline Reference & 10 , this study & 9 & 9 \\
\hline
\end{tabular}

are not found in both LMG 18311 and CNRZ1066 (Additional file 3). These ORFs are located in 27 chromosomal loci, six of which spanned more than $8 \mathrm{~kb}$ in length (Figure 2). Interestingly, the distribution of these 27 loci appears to concentrate at regions distal from the origin of DNA replication (Figure 1, circle 12). Remarkably, 14 of these 27 LMD-9 specific chromosomal loci are flanked by intact or truncated transposases, suggesting IS elements as a significant contributor to the genome diversity in S. thermophilus.

Deduced proteins from 57 (45\%) of the 127 LMD-9 specific ORFs have no orthologous counterpart in other streptococcal species (see below), indicating they likely originated via lateral gene transfer (LGT). Approximately one-fifth (24/127) of the LMD-9 specific ORFs are predicted pseudogenes, providing further evidence that gene decay had continued following strain divergence. The majority of the remaining intact genes (40/103 or 39\%) encode for proteins involved in amino acid transport and metabolism, transposases, or proteins having unknown functions.

Eight of the LMD-9 specific regions contain ORFs with conserved homologs in Streptococcus salivarius (more than $90 \%$ sequence identity), notably genes coding for glycosyltranferases (R12 and R20), the his gene cluster (R14), and numerous unknown proteins, suggesting that these ORFs were most likely lost in both LMG 18311 and CNRZ1066 strains. Similar to both LMG 18311 and CNRZ1066, genes implicated in virulence from the pathogenic streptococci are either absent or truncated in the LMD-9 genome, or are involved in other physiological functions relevant to the adaptation towards dairy environment. In addition, only 17 of the LMD-9 specific ORFs have closest orthologs with the pathogenic streptococci.

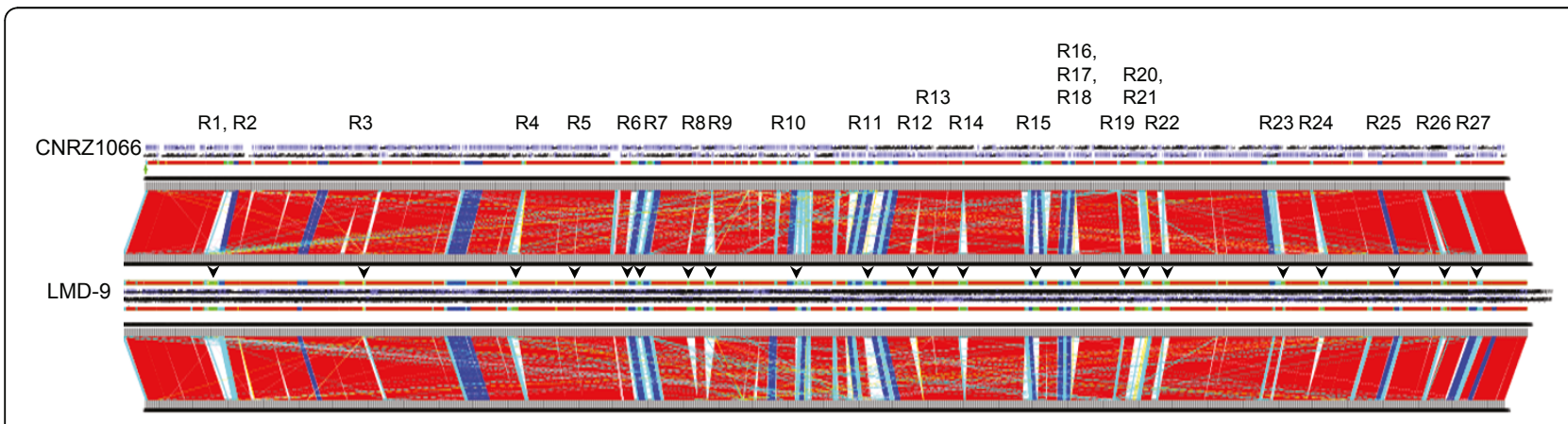

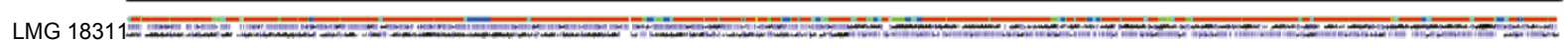

Figure 2 Alignment of $\boldsymbol{S}$. thermophilus genomes. Genome alignment of S. thermophilus LMD-9 with CNRZ1066 and LMG 18311 strains was performed using the GenomeComp software tool [32]. Alignment lengths are color-coded as follows: red, >10,000 bp; blue, 5,000 to 10,000 bp; cyan, 1,000 to 5,000 bp; yellow, 500 to 1,000 bp, and pink, <500 bp. Unique regions are in green. LMD-9 specific regions are indicated with black arrowheads above the LMD-9 linear chromosome, with region designation shown at the top of the upper canvas. ORFs present within these regions are described in Additional file 3. 
Most of these genes encode for unknown proteins and transporters, some of which are truncated. One region (R9) encoding a partial spermidine/putrescine ABC transporter, along with a putative chloride channel and a cell envelope serine proteinase PrtS, is flanked by transposases and showed conserved sequence and synteny to Streptococcus suis [39]. While the absence of PrtS in both LMG 18311 and CNRZ1066 strains has been previously noted [16], this region, a so-called "ecological island", was frequently found in the chromosomes of recently emerged $S$. thermophilus industrial strains [39].

Three regions ( $22, R 5$, and R11) encode for proteins related to exopolysaccharides (EPS) biosynthesis with closest identities to corresponding proteins from Enterococcus faecalis (STER0149, STER0150), Lactobacillus rhamnosus (STER0656), Lactobacillus gasseri (STER1061), Lactococcus lactis (STER1057), Clostridium beijerinckii (STER1059), and intriguingly, gastrointestinal-specific bacteria Eubacterium rectale and Ruminococcus obeum (STER1063-1065). Aside from the aforementioned PrtS, LMD-9 also acquired an additional dipeptide/oligopeptide $\mathrm{ABC}$ transporter (STER0142-STER0145) located within R2 that potentially contributes to nutrient uptake in milk environments. This peptide transporter exhibited moderate similarity (50 to $60 \%$ identity) to the corresponding transporter components from various Bifidobacterium species also specifically associated with the human gut. Eight other LMD-9 specific regions encode for proteins that potentially broadened its repertoire of defense mechanisms. These included two copies of a putative antimicrobial peptide $\mathrm{ABC}$ transporter (STER $40571-0572$ and STER1347-1348) that have close orthologs in Leuconostoc mesenteroides and Lactobacillus fermentum. Also identified were components of restriction-modification (R/M) systems (STER0731 and STER0750), an abortive infection phage resistance protein (STER1698), and the previously identified CRISPR3 region (R22) [18] which showed moderate sequence homology (60 to $87 \%$ identity) to orthologs in Streptococcus mutans, Streptococcus agalactiae, Streptococcus dysgalactiae, Streptococcus equi, and Streptococcus pyogenes. Strain LMD-9 also encodes a 1,009-amino acid residues surface protein in R4 (STER0576) that was closely related to mucus-binding (MucBP) domain-containing proteins in lactobacilli. This putative mucus-binding protein (Mub) is not found in other streptococci, and has a single MucBP domain with an N-terminal YSIRK signal peptide and a C-terminal LPNTG cell wall anchoring motif.

The genome of LMD-9 harbors a prophage remnant (STER0810-0830) where part of the region (STER08160830) showed high degree of conservation with the prophage remnant in CNRZ1066. Examination of the corresponding chromosomal region in LMG 18311 and S. salivarius SK126 draft genome, both of which lack the phage remnant, indicated that the prophage elements in both LMD-9 and CNRZ1066 were integrated between the genes encoding for para-aminobenzoate synthases component I (STER0809/str0771) and 5-methyltetrahydropteroyltriglutamate-homocysteine S-methyltransferase (STER0831/str0785). The remaining $3.7 \mathrm{~kb}$ of the prophage region (R8), which is present only in LMD-9, exhibited a high degree of sequence homology with lactococcal phages, suggesting the acquisition of this region after strain divergence.

\section{(ii) Regions absent in LMD-9}

Approximately $105 \mathrm{~kb}$ and $103 \mathrm{~kb}$ in total of chromosomal regions in CNRZ1066 and LMG 18311, respectively, were absent in the LMD-9 genome. Overall, 57 ORFs interspersed in 14 chromosomal regions were present in both strains but not in LMD-9. Fifteen of these ORFs are pseudogenes, including remnants of gene clusters coding for an amidase (str/stu0973), an amino acid ABC transporter substrate-binding protein (str/stu0975), and a maltose/maltodextrin ABC transporter (str/stu1015strstu1017). These genes appear intact in S. salivarius and $S$. mutans, indicating that like LMD-9, these genes are also undergoing degeneration, albeit at a slower rate, in the other two strains. On the other hand, as in Streptococcus gordonii, both CNRZ1066 and LMG 18311 possessed a second copy of biotin synthase-encoding gene bio $Y 2(\mathrm{str} / \mathrm{stu} 1308)$ in a $8.6-\mathrm{kb}$ unique region ( $\mathrm{str} /$ stu1308-1315), which displayed $42 \%$ sequence identity to bioY1 present in all three $S$. thermophilus strains. The remainder of the region encodes for a proline/glycine ABC transporter that showed $60-70 \%$ similarity to the corresponding orthologs in Streptococcus gallolyticus, and a partial L-histidine degradation pathway consisting of histidine ammonia-lyase ( $h u t H$, str/stu1313; EC 4.3.1.3) and a urocanate hydratase (hut $U$, str/stu1315; EC 4.2.1.49). These enzymes are involved in the initial steps of the pathway, although both CNRZ1066 and LMG 18311 lack imidazolonepropionase (EC 3.5.2.7) and formiminoglutamase (EC 3.5.3.8) required to yield the final product, L-glutamate. Both strains also appeared to have acquired type III R/M system components (str/stu 0884 and 0885) that most closely resembled the orthologs in Listeria monocytogenes (70 to $80 \%$ identity). Strain LMD-9 also lacks genes encoding two oxidative stress defense proteins (str/stu0183 and str/stu1314) in CNRZ1066 and LMG 18311 that are homologous (99\% identity) to the OsrE and OsrF of $S$. thermophilus CNRZ368 [40].

\section{Genome comparison of S. thermophilus with other streptococci}

Comparative analysis of the ORFeomes among sequenced streptococci using the FGD analysis revealed functional clustering of the species that is consistent 
with their grouping (pyogenic, mutans, salivarius, and mitis) based on 16S rDNA phylogenetic inferences [41] (Figure 3). Both S. thermophilus CNRZ1066 and LMG 18311 formed a tight cluster that diverges from the LMD-9 strain. The S. thermophilus strains and the other member of the salivarius group, S. salivarius SK126 (a human skin isolate), formed a functional cluster, with $S$. mutans as the closest pathogenic relative. Comparison of genome content between all three S. thermophilus strains and the draft genome of S. salivarius SK126 revealed that both species shared approximately $75 \%$ of the protein-coding genes (E-value cutoff 1e-10). A set of 68 genes was identified as unique for $S$. thermophilus compared to SK126, including genes encoding urease biogenesis, lactose utilization (lactose permease LacS and beta-galactosidase LacZ), CRISPR elements, transposases, various transporters, a type II R/M system, and numerous hypothetical proteins of unknown function (Additional file 4). It is interesting to note that the urease gene cluster, lacS and lac $Z$ are commonly found in human oral-associated strains of $S$. salivarius but apparently are absent in the skin-associated SK126 strain. Since these genes are not universally present in other streptococci, it is plausible that they were acquired by clonal populations of $S$. salivarius and S. thermophilus via LGT in their natural habitats. Meanwhile, 428 genes from S. salivarius SK126 are absent in all three $S$. thermophilus strains. S. salivarius has a broader repertoire of genes involved in carbohydrate utilization and biosynthesis of complex polysaccharides, including an elaborate set of glucosyltransferases for glucan biosynthesis, as well as the complete pathway for the synthesis of glycogen (STRSA0001_0011 to STRSA0001_0014). In addition to the SrtA sortase, which is present as an intact gene only in LMD-9 among the sequenced $S$. thermophilus strains, S. salivarius also possesses an SrtB sortase, and a wider array of cell surface proteins.

Among all the sequenced streptococcal strains included in the comparative analysis, 510 genes were found conserved (E-value $\leq 1 \mathrm{e}-60$ cutoff) in all strains. These core genes, which are mostly involved in housekeeping functions, account for $27.8 \%$ of the total ORFs encoded in the LMD-9 chromosome. Among the non-core genes, only 13 have no significant identity to all other streptococci and therefore are species-specific for S. thermophilus, which includes a putative arginase/agmatinase, a homoserine/ threonine efflux protein, a probable serine/threonine protein kinase, oxidoreductases, transposases and conserved hypothetical proteins (Additional file 4). Meanwhile, 8 genes were identified as conserved in all streptococci excluding S. thermophilus, notably genes that encode for enzymes from the tagatose-6-phosphate pathway, a transaldolase (EC 2.2.1.2) involved in the pentose phosphate pathway, and a carbamate kinase (EC 2.7.2.2) belonging to the arginine deiminase pathway.

Recent extensive genome analyses of pathogenic streptococci have established that capsular polysaccharide genes and phage elements are major contributors to the genome diversity among strains of Streptococcus pneumoniae [42,43] and S. pyogenes [44-48], respectively. Our comparative genome analysis revealed functional sub-clusterings among the sequenced strains of S. pneumoniae and S. pyogenes (Figure 3). Within the S. pneumoniae strains, eleven genes were detected as unique for both R6 and D39 (cluster SP1), notably genes encoding for capsular polysaccharide biosynthesis (Additional file 5). On the other hand, the SP1 strains lack seven genes that are conserved in all cluster 2 (SP2) strains of S. pneumoniae. Aside from capsular polysaccharide biosynthetic genes, both clusters also differ by the exclusive presence of a bacteriocin immunity protein $\mathrm{BlpX}$ and a prophage maintenance system killer protein in the SP2 cluster strains. It was previously reported in the TIGR4 (an SP2 strain) genome that the bacteriocin biosynthesis and immunity protein genes are among those that underwent lineage-specific gene duplication [42]. Meanwhile, the $S$. pyogenes strains appeared as three subclusters, with an apparent divergence of the M1 strain SF370 (subcluster SY1) from the other strains, including the contemporary M1 strain MGAS5005. Only four genes, Spy0942, Spy0944, Spy0978 and Spy2147, were detected as SF370-specific, of which the latter three are phage-associated proteins (Additional file 6). Interestingly, no ORF was detected as conserved in all other $S$. pyogenes strains that is absent in SF370. The major difference between the remaining SY2 and SY3 subclusters is the presence of lantibiotic biosynthetic genes, phagerelated genes, and virulence-associated genes, such as fibronectin-binding protein and serum opacity factor in the SY2 subcluster.

\section{Metabolic and biosynthetic capabilities}

The LMD-9 genome encodes sets of enzymes conserved in the LMG 18311 and CNRZ1066 strains for glycolytic pathway, the non-oxidative branch of the pentose phosphate pathway, and pyruvate metabolism [16]. Unlike other closely-related Gram-positive species of lactococci, enterococci, bacilli, listeria and lactobacilli, the pyruvate carboxylase-encoding gene required for the formation of oxaloacetate from pyruvate is absent in all streptococci. Instead, the key precursor for the biosynthesis of aspartate family amino acids is generated from phosphoenolpyruvate by a phosphoenolpyruvate carboxylase (Ppc) conserved in the streptococci. In S. thermophilus, it was demonstrated that the activity of Ppc is essential for the bioavailability of L-aspartic acid during growth in milk [49]. 


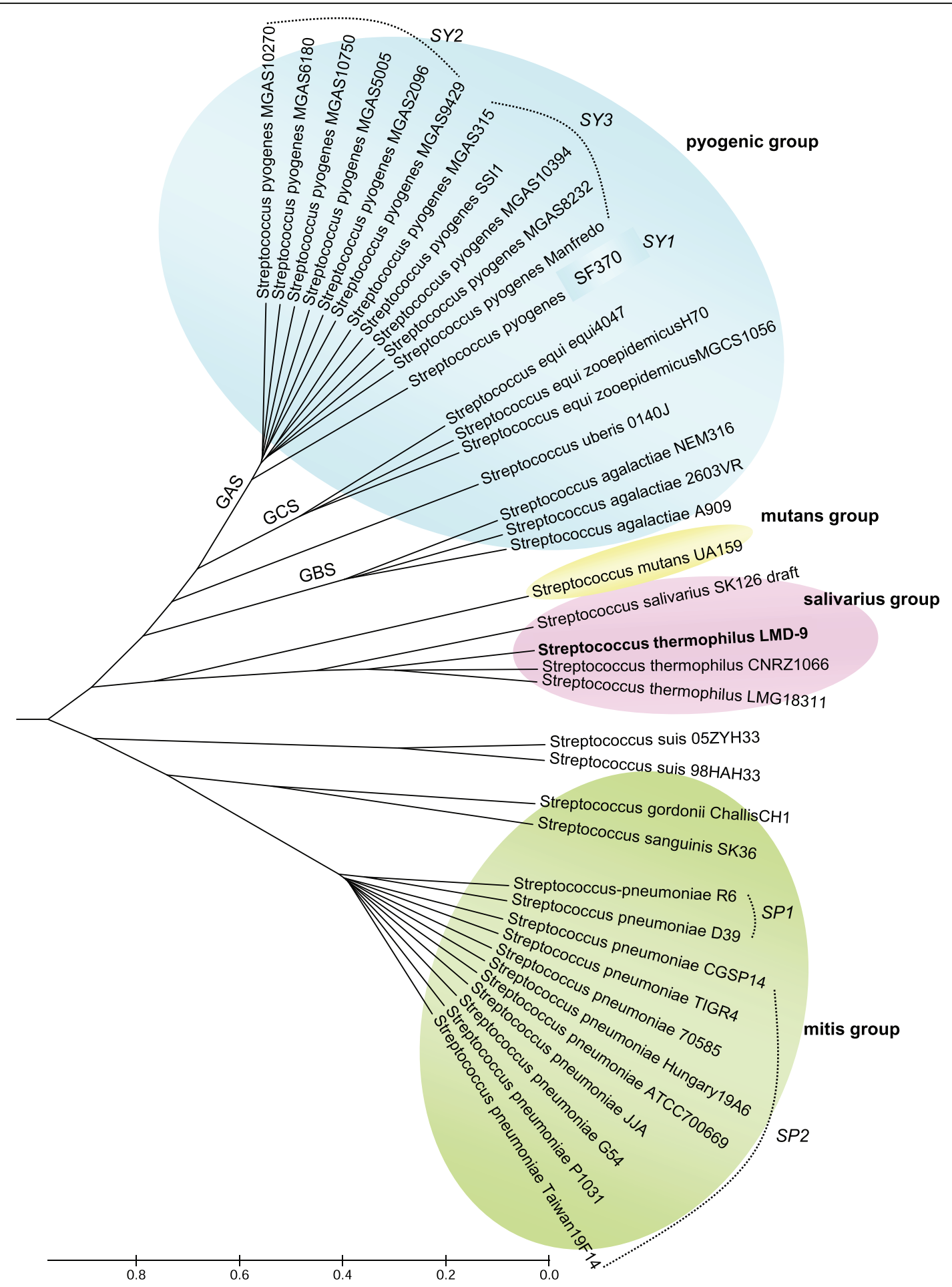

Figure 3 Functional genome distribution (FGD) tree of 39 Streptococcus genomes. FGD analysis of 39 Streptococcus strains for which genome sequences are available, depicting the functional relationship among the species and strains based on the amino acid sequence similarities of the predicted ORFeomes. S. thermophilus strains formed a functional cluster with S. salivarius as the closest relative. The FGD analysis also predicted functional sub-clusterings among the S. pneumoniae and S. pyogenes strains. Approximated branch lengths, representing the level of functional similarity between genomes are depicted by distance units (du). 
Unlike its pathogenic counterparts such as $S$. agalactiae, S. pyogenes and S. pneumoniae, which are auxotrophic for most amino acids, the putative pathways for the biosynthesis of all amino acids with the possible exception of alanine and lysine were identified in the LMD-9 genome. The absence or inactivation of these enzymes will also affect peptidoglycan biosynthesis, which suggests that the generation of key intermediates (L-alanine and meso-diaminopimelate) for peptidoglycan synthesis may involve an alternate pathway. LMD-9 has a 7.4-kb his gene cluster (STER1198 to STER1207) that encodes a complete histidine biosynthetic pathway, but appears to be deleted in LMG 18311 and CNRZ1066 [16], which is further evidenced by the relative conservation of the adjacent genes flanking the his cluster between LMD-9 and S. salivarius SK126. Nonetheless, in LMD-9, we speculated that the presence of several copies of transposases at the upstream vicinity of the his gene loci might be involved in the inactivation of an EPS biosynthesis protein homolog ( $\psi$ STER1211) and the deletion of glycosyltransferase genes which are otherwise found intact in the corresponding region in S. salivarius.

S. thermophilus is predicted to be capable of de novo synthesis of both purine and pyrimidine nucleotides. Like the majority of the streptococci, $S$. thermophilus has the gene which encodes for nucleoside diphosphate kinase (EC 2.7.4.6; STER0929), a key enzyme for generating dTTP, which was not found in S. mutans [50], S. sanguinis [51], S. gordonii, and S. pyogenes. Genes encoding salvage enzymes were identified for the utilization and recycling of pre-formed nucleobases and nucleosides for nucleotide synthesis as well as sources of energy, carbon, and nitrogen. Purine salvage enzymes include adenine phosphoribosyltransferase (STER1190), hypoxanthine phosphoribosyltransferase (STER0013), and purine nucleoside phosphorylases (STER1072 and STER1075) allow salvage of adenine, guanine, and xanthine nucleobases. Meanwhile, genes encoding for a xanthine phosphorybosyltransferase and a putative xanthine permease ( $\psi$ STER1311 and $\psi$ STER1312) that are present as a potential operon appears to be inactivated by frameshifts. The pyrimidine salvage pathway involves uracil phosphoribosyltransferase (STER0394) that converts uracil to UMP, which is complemented by two putative uracil uptake permeases (STER0375 and STER0556). The pyrimidine-nucleoside phosphorylase (EC 2.4.2.2) gene responsible for salvaging cytosine is truncated in all three S. thermophilus strains, whereas the gene is intact in the closely related $S$. salivarius and $S$. mutans. Nonetheless, the presence of a uridine kinase (STER1238) and a thymidine kinase (STER0792) potentially permits uridine, cytidine, and thymidine nucleosides as additional substrates for nucleotide synthesis.
While the biosynthetic pathways for the cofactors biotin, pantothenate, pyridoxine, thiamine, and riboflavin were incomplete, it is predicted that $S$. thermophilus is capable of synthesizing coenyzme A (CoA) and acyl carrier protein with L-cysteine or pre-formed pantothenate as precursors. As previously noted by van de Guchte $e t$ al. [52], the organism also possesses the complete pathway for folate biosynthesis from GTP or $p$-aminobenzoic acid (PABA) precursor, as well as de novo synthesis of PABA from chorismate (STER1554, STER0809).

\section{Protein secretion systems}

The transport of proteins across the cytoplasmic membrane is a function of the general secretion (Sec) pathway. A second pathway determined as the TAT (twin arginine translocation) pathway secretes folded proteins. LMD-9 encodes components of both pathways that have been described in both LMG 18311 and CNRZ1066 [16]. As with the YidC orthologs in LMG 18311 and CNRZ1066, these proteins may interact with SecYEG, the membranespanning translocase, thereby potentially playing a role in membrane protein folding and assisting SecYEG to insert membrane proteins into the membrane [53].

The TAT translocase consists of either two, TatA and TatC, or three TatA, TatB and TatC integrated membrane components with the required energy provided by the proton motive force [54]. The TatP prediction tool was used to search for proteins encoded by LMD-9 that have a TAT signal peptide and TAT motif [30]. Seventeen proteins were identified, but only STER1023 has both a TAT signal peptide and TAT motif (Additional file 7), whereas the remaining 16 contained a potential TAT signal peptide only. STER1023 encodes for a probable iron-dependent peroxidase that may play a role in tolerance to oxidative stress and shows high identity (97\%) to the corresponding ortholog in S. salivarius. BLAST analysis with the nonredundant database revealed orthologs in S. salivarius SK126 with high identity ( 94\%) for TatA and TatC. Orthologs for both TatA and TatC were found in the oral commensal and also opportunistic pathogenic streptococci S. gordonii, Streptococcus mitis, S. sanguinis and Streptococcus parasanguinis indicating that the TatAC rather than Tat $\mathrm{ABC}$ system is more common in streptococci.

\section{EPS gene clusters}

Similar to S. thermophilus CNRZ1066 and LMG 18311, LMD-9 has two EPS clusters, an eps cluster and a rgp cluster, the latter of which resembled the rgp cluster of $S$. mutans that encodes for the biosynthesis of rhamnose-glucose polysaccharide [16]. As in the case for most S. thermophilus eps operons [55], the eps clusters in all three S. thermophilus and S. salivarius SK126 showed conserved sequence ( $\geq 90 \%$ identity) and synteny 


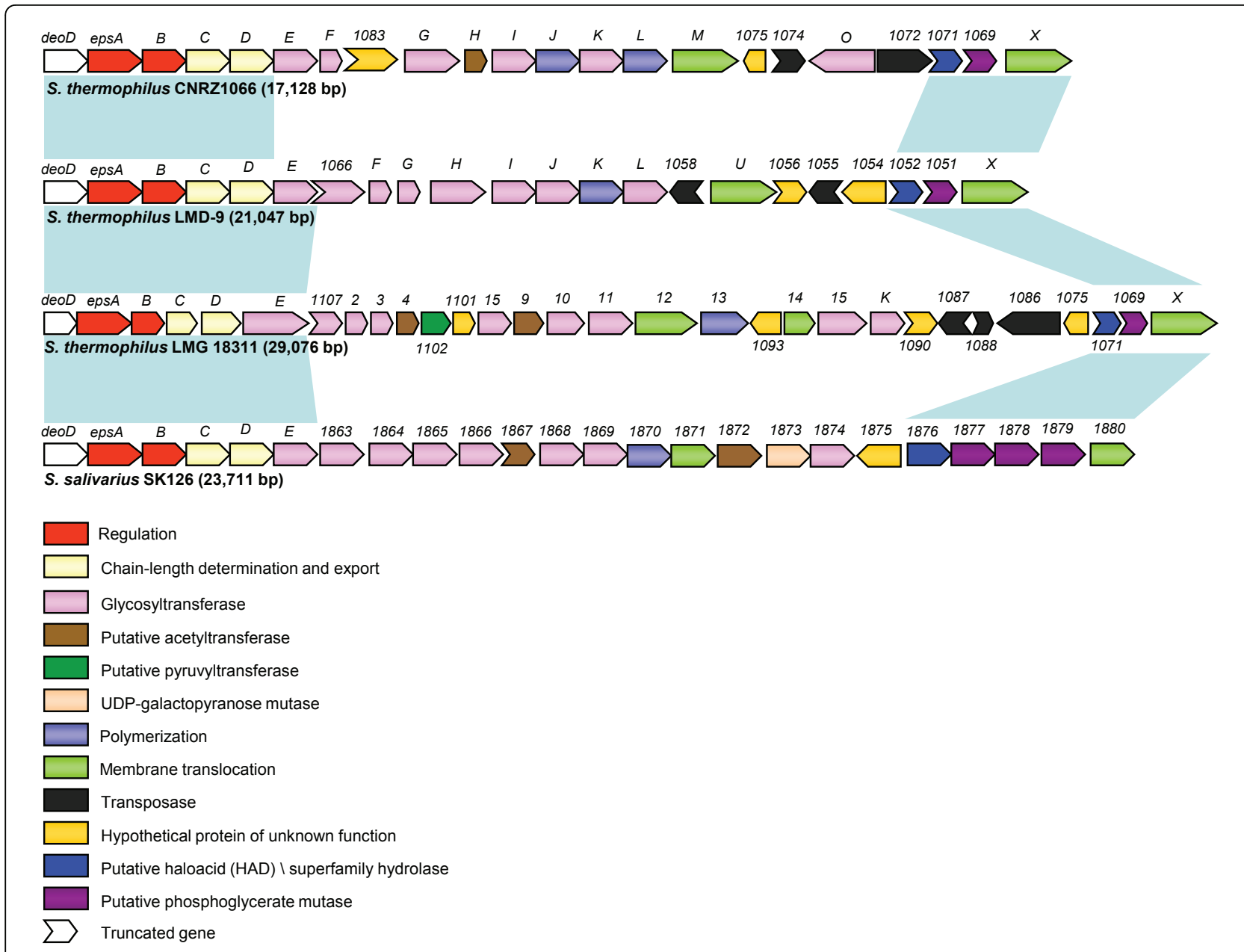

Figure 4 The exopolysaccharide (EPS) biosynthetic gene clusters in S. thermophilus and S. salivarius SK126. ORFs were color-COded according to their prediction functions (see legend at lower left panel). Regions exhibiting sequence similarities among the eps clusters are shown in light blue-shaded boxes. The size of each eps cluster is indicated in bracket.

confined to the terminal 5' and 3' regions of the clusters (Figure 4). All four eps clusters are flanked by deoD (encoding a purine nucleoside phosphorylase) and $b g l H$ gene (encoding a beta-glucosidase), noting that $b g l H$ is interrupted by frameshifts in all three $S$. thermophilus strains.

There is considerable variation in the sequence and little synteny in the central regions of the eps clusters in the $S$. thermophilus strains. Interestingly, the EPS biosynthetic components within this region of LMD-9 exhibited highest sequence similarity to the corresponding proteins in dairy associated L. lactis as well as microorganisms of human intestinal origin, such as E. rectale, $R$. obeum, and L. gasseri (R11, Additional file 3). The presence of two divergently-orientated transposase remnants together with the atypically low $\mathrm{G}+\mathrm{C}$ content indicates that this region is likely acquired via LGT. Approximately $10 \mathrm{~kb}$ larger, the LMG 18311 eps cluster has a more complex mosaic structure, with several of the genes conserved with the eps genes from S. thermophilus IP6757. Meanwhile, CNRZ1066 has the most conservative eps cluster that is almost identical to the ones previously described in S. thermophilus MTC360, MR-2C and Sfi6 [56]

Although the physiological function of EPS production in S. thermophilus remains elusive, the marked variability of the eps regions and prominent presence of mobile elements at the vicinity of the eps clusters suggests the presence of unknown factors that drives the evolution and diversification of EPS among S. thermophilus strains via lateral genetic exchange. Aside from the main contribution of EPS to the texture and rheology of fermented products, EPS has also recently been associated with host immunostimulative effects [57]. It is likely that the diversity of the eps clusters may impart distinct immunomodulatory characteristics of the EPS produced by S. thermophilus. 
Specialized adaptation to the dairy environment. (i) Carbohydrate utilization

Lactose represents the principal carbohydrate component in milk. Like the majority of the S. thermophilus strains [58], LMD-9 was only able to ferment lactose, sucrose, and glucose, in order of preference, as assessed with API test and growth experiments (data not shown). Major gene decay among carbohydrate utilization genes occurred in LMD-9, a similar observation that was previously reported for strains LMG 18311 and CNRZ1066 [9]. In fact, the sets of intact carbohydrate utilization genes and pseudogenes are almost identical to those of LMG 18311 and CNRZ1066 (Table 2), indicating that gene degeneration occurred in the ancestor strain. Consequently, the limited repertoire of carbohydrate metabolic genes include three intact sugar hydrolases: a betagalactosidase, a sucrose-6-phosphate hydrolase, and an intracellular alpha-amylase, in addition to (i) three intact PTS with predicted specificities for mannose/fructose, cellobiose, and sucrose; (ii) three putative sugar $A B C$ transporters of unknown substrates, and (iii) two sugar permeases predicted for the uptake of glucose and lactose, respectively. The glucose/ribose permease (STER0891) gene in all three S. thermophilus strains is absent in S. salivarius SK126 and is located within a previously identified 17-kb LGT hot spot [9]. This sugar permease showed high identity (88 to $91 \%$ ) to the orthologs in L. rhamnosus, L. lactis and L. helveticus that shared a close ecological niche. Meanwhile, all three $S$. thermophilus possessed complete pathways for the conversion of $\mathrm{N}$-acetylglucosamine to substrates of glycolysis, amino acid metabolism and peptidoglycan biosynthesis. Bovine milk contains oligosaccharides, glycoproteins, and glycolipids that potentially serve as a source for $\mathrm{N}$-acetylglucosamine. This suggests that aside from lactose, $S$. thermophilus is also equipped to utilize $\mathrm{N}$-acetylglucosamine as an energy source during growth in milk.

The gal-lac gene clusters are conserved in S. thermophilus and S. salivarius [59], and analogous lac-gal gene

Table 2 Carbohydrate transport systems and sugar hydrolases in S. thermophilus LMD-9

\begin{tabular}{|c|c|c|c|}
\hline Locus Tag & Encoded protein & Homolog in LMG 18311 & Homolog in CNRZ1066 \\
\hline \multicolumn{4}{|c|}{ Phosphotransferase system (PTS) } \\
\hline STER0370 & mannose/fructose PTS ॥D & stu0331 & str0331 \\
\hline STER0371 & mannose/fructose PTS IIC & stu0332 & str0332 \\
\hline STER0372 & mannose/fructose PTS $\| A B$ & stu0333 & str0333 \\
\hline STER1323 & Cellobiose PTS IIC & stu1368 & $\operatorname{str1368}$ \\
\hline STER1710 & Sucrose PTS ElIBCA & stu1734 & str1734 \\
\hline ४STER0237 & glucose/N-acetylglucosamine PTS EIICBA, trunc. & stu0189 & str0189 \\
\hline$\psi$ ४STER0446 & Fructose PTS IIA, trunc. & stu0401 & str0401 \\
\hline$\psi$ ४STER0544 & Beta-glucoside PTS EIIABC, trunc. & stu0512 & str0512 \\
\hline$\psi$ STER1862 & Trehalose PTS EIIABC, trunc. & stu1890 & $\operatorname{str} 1890$ \\
\hline \multicolumn{4}{|c|}{$A B C$ transporter } \\
\hline STER0856 & ABC transporter, substrate-binding protein & stu0808 & str0808 \\
\hline STER0857 & Sugar ABC transporter, ATPase component & stu0809 & str0809 \\
\hline STER0858 & Sugar $A B C$ transporter, permease component & stu0810 & str0810 \\
\hline STER0859 & Sugar $A B C$ transporter, permease component & stu0811 & str0811 \\
\hline STER1153 & ABC transporter, ATPase component & stu1188 & $\operatorname{str} 1188$ \\
\hline STER1154 & Ribose/xylose/arabinose $\mathrm{ABC}$ transporter, permease component & stu1189 & $\operatorname{str1189}$ \\
\hline STER1155 & $A B C$ transporter, substrate-binding protein & stu1190 & str1190 \\
\hline$\psi$ ४STER0249 & Ribose/xylose/arabinose $\mathrm{ABC}$ transporter, permease component, trunc. & stu0201 & str0201 \\
\hline \multicolumn{4}{|c|}{ Sugar permease } \\
\hline STER0891 & Glucose/ribose permease & stu0855 & str0855 \\
\hline STER1367 & Lactose permease, LacS & stu1398 & str1398 \\
\hline \multicolumn{4}{|c|}{ Sugar hydrolase } \\
\hline STER1366 & Beta-galactosidase, LacZ & stu1397 & str1397 \\
\hline STER1500 & Alpha-amylase (cytoplasmic) & stu1542 & str1542 \\
\hline STER1711 & Sucrose-6-phosphate hydrolase & stu1735 & str1735 \\
\hline ४STER1029 & Putative pullulanase, trunc. & & \\
\hline$\psi$ ४STER1049 & Beta-glucosidase, trunc. & stu1064 & str1064 \\
\hline$\psi$ ४STER1594 & 6-phospho-beta-glucosidase, trunc. & stu1631 & str1631 \\
\hline$\psi$ ४STER1861 & Trehalose 6-phosphate hydrolase, trunc. & stu1888 & $\operatorname{str} 1888$ \\
\hline
\end{tabular}


clusters are also present in the sequenced strains of L. lactis, Lactobacillus acidophilus, Lactobacillus johnsonii, Lactobacillus plantarum and Lactobacillus salivarius. However, L. bulgaricus, a companion culture for $S$. thermophilus during yogurt fermentation, only has a lacSZ operon. While absent in S. pyogenes and Streptococcus uberis, the galRKTE gene cluster is relatively conserved in the other sequenced streptococcal species. The galM gene encoding an aldose 1-epimerase is found only in strains of $S$. thermophilus, S. salivarius and S. equi. The GalM orthologs from $S$. equi strains, however, showed only moderate sequence homology $(50 \%$ sequence identity) with the corresponding proteins from the former two species.

Orthologs of the S. thermophilus cytoplasmic alphaamylase were found in most sequenced streptococci (with 60-70\% sequence identity) with the exception of S. pyogenes and S. suis strains. Due to the inability of LMD-9 to ferment starch, the intracellular alpha-amylase is less likely to have a direct role in starch degradation, as previously described for Streptococcus bovis and S. mutans. The intracellular amylases of S. bovis strains were shown to be essential for rapid cell growth [60], or acted as maltotriose-producing endo-amylase [61]. In $S$. mutans, this enzyme is involved in the accumulation of glycogen-like intracellular polysaccharide during growth on maltose [62]. Meanwhile, remnants of a putative pullulanase, a trehalose 6-phosphate hydrolase, and two betaglucosidase-encoding genes are also present in the genome, the latter of which likely explained the inability of LMD-9 to utilize cellobiose despite the presence of an intact orphan cellobiose PTS EIIC-encoding gene. Overall, the majority of the pseudogenes are involved in the transport and hydrolysis of glucosides, suggesting a possible niche transit of $S$. thermophilus ancestor strain from plant to dairy environment.

\section{(ii) Proteolytic system}

Milk is poor in free amino acids as its rich protein content is present mainly in the form of casein. Although LMD-9 is predicted to be capable of synthesizing most of the amino acids, its genome encodes an intact cell wall-associated proteinase PrtS, an abundance of cytoplasmic peptidases and transport systems that allows the utilization of exogeneous protein source in milk. As previously mentioned, the PrtS is encoded in one of the LMD-9 unique regions (R9; Additional file 3) and is highly conserved (95\% identity) with the PrtS of S. suis. Although recent work suggested that PrtS contributes to the virulence of S. suis [63], it is well established that in $S$. thermophilus strains, the primary role of PrtS involved the cleavage of casein to oligopeptides, a clear function related to its dairy adaptation. Previous study with the PrtS of S. thermophilus CNRZ385 showed that the proteinase is essential for growth in milk unless co-cultured with L. bulgaricus [64].

The LMD-9 genome possessed all 12 cytoplasmic peptidases (pepA, рерC, pepF, рерM, pеpN, рерO, pepP, pepQ, pepS, pepT, pep $V$, pepX) present in both LMG 18311 and CNRZ1066 [16] strains that potentially provide flexibility in peptide substrate specificities. Unlike most lactic acid bacteria where some peptidase genes formed operon structures with adjacent amino acid/peptide transporter genes, none of the peptidase-encoding genes in S. thermophilus is located at the vicinity of any amino acid/peptide uptake system. Nine complete ABC transport systems are complemented by five permease porters for the uptake of amino acids, oligopeptides and polyamines, including: two oligopeptide opp ABC transporters (STER0142-0145, STER1405-1409), three polar amino acids ABC transporters (STER0905-0907, STER1539-1542, STER1617-1619), one ABC transporter (STER0398-0402) and two symporters for branched-chain amino acids (STER0993, STER1315), one glutamine ABC transporter (STER14611462), and one spermidine/putrescine $A B C$ transporter (STER1493-1496). As mentioned previously, one of the opp transport systems (STER0142-0145) is located within a 10.2-kb unique region (R2; Additional file 3) of LMD-9 that shared moderate homology with distant species of Bifidobacterium. This finding has led us to speculate that LGT events potentially occurred beyond the milk niche.

\section{(iii) Stress response and transcriptional regulation}

S. thermophilus often encounter physiological stresses caused by adverse conditions during commercial production of yogurt and cheeses as well as preservation of starter cultures. These include low $\mathrm{pH}$, changes in osmolarity and temperatures, oxidative stress, nutrient limitation and competition in mixed culture environments. The ability of strains to adapt and perform in these industrial stress conditions is crucial to the consistency and organoleptic properties of the final products. The LMD-9 genome encodes a proton translocating $\mathrm{F}_{0} \mathrm{~F}_{1}$-ATPase system (STER0515-0522) and a putative cation transport ATPase (STER1107) that likely contribute to internal pH homeostasis in high acidity environments. To cope with oxidative stress, LMD-9 possesses a Mn-superoxide dismutase (STER0762) for the elimination of reactive oxygen species along with disulfide-reducing pathway enzymes, including a glutathione reductase (STER0447), thioredoxins (STER1776, STER1825) and thioredoxin reductases (STER1382, STER1615). Other putative proteins involved in oxidative stress tolerance include an NADH oxidase (STER1258) that potentially plays a role in the removal of oxygen and two methionine sulfoxide reductases (STER1314 and STER1596) that serve housekeeping protein function via methionine sulfoxide reduction in proteins. 
The classical class I heat-shock genes encoding the conserved chaperone complexes GrpE-DnaK-DnaJ and GroES-GroEL were identified, with the HrcA repressorencoding gene located upstream of the grpE-dnaK-dnaJ operon. Other heat-shock genes include the class III CtsR repressor (STER0108), along with four Clp-ATPase chaperones (STER0109, STER0625, STER0648, STER1578) and a ClpP proteolytic component (STER0395) that degrade nonfunctional proteins. The pSTER_B plasmid of LMD-9, a pC194 family rolling circle replicating plasmid, encodes a 143-amino acid small heat shock protein (sHsp; pSTER_B1) that is conserved ( $\geq 81 \%$ identity) in several other plasmid-bearing $S$. thermophilus strains $[65,66]$. In fact, pSTER_B1 is identical to the sHsp from pER1-1 of $S$. thermophilus ER1 along with the upstream conserved CtsR regulatory region [65], and the RepA deduced proteins from both plasmids shared 99\% sequence identity. Expression of these plasmid-encoded sHsp has been shown to be upregulated at low $\mathrm{pH}$ or elevated temperatures, suggesting that PSTER_B potentially provides multitude cross-protections that enhance the survival of LMD-9 under various stress conditions. Meanwhile, S. thermophilus has two cold-shock protein genes (STER0879 and STER0880) arranged in tandem and both deduced proteins shared only $50 \%$ identity. No homolog of STER0879 was found among other streptococci, whereas STER0880 is conserved mainly among the pyogenic group of streptococci ( 80\% identity). Interestingly, STER0879 and STER0880 showed highest similarity to the tandem $\operatorname{csp} C D$ genes in L. lactis (95\% identity), which was induced by cold shock at $10^{\circ} \mathrm{C}$ [67]. While one glycine-betaine transporter, OpuD (STRSA0001_1242) was found in the S. salivarius SK126 draft genome, no specific transporter or biosynthetic pathway for compatible solutes was detected in the S. thermophilus genomes. Nevertheless, several Trktype $\mathrm{K}^{+}$transporter homologs are present (STER0311STER0312 and STER0350-STER0351) that may play a role in cytoplasmic osmoregulation.

The ability of microorganisms to survive and adapt to changing environments relies on the intricate sensing and signal transduction mechanisms of two-component regulatory systems. Hols et al. [16] previously noted that all three $S$. thermophilus sequenced strains have the same set of 20 two-component regulatory system (2CRS) genes. Closer examination of the LMD-9 2CRS gene sets revealed six sets of 2 CRS consisting of both histidine protein kinase $(\mathrm{HK})$ and response-regulator (RR) genes adjacent to each other (Additional file 8), compared to seven and eight sets of 2CRS that were identified in CNRZ1066 and LMG 18311 strains, respectively. In comparison, the S. salivarius SK126 draft genome has 12 sets of HK-RR systems, reflecting its adaptation to a more complex lifestyle.

\section{(iv) Cell surface proteins}

Cell surface proteins can be anchored to the cell wall peptidoglycan through the action of sortase which recognizes an LPXTG binding motif preceding a hydrophobic region at the $\mathrm{C}$-terminus of the precursor proteins. The LMD-9 strain encodes for an intact sortase, SrtA (STER1255) which presents as a pseudogene in both LMG 18311 and CNRZ1066 [16]. In addition, three proteins with cell sorting motifs are pseudogenes in LMG 18311 and CNRZ1066, whereas LMD-9 encodes for three sortase-dependant proteins in addition to two pseudogenes containing the sortase recognition motif. The three intact proteins which all contain an LPNTG motif are the aforementioned PrtS and Mub, in addition to a cyclo-nucleotide phosphodiesterase CpdB (STER0198). Inactivation of the srtA gene in S. sanguinis [68] and S. uberis [69] demonstrated its role in bacterial colonization. The sortase from LMD-9 showed high identity $(\sim 90 \%)$ with the sortase from S. salivarius SK126. In addition to a second sortase, SrtB, S. salivarius also encodes for 420 proteins with signal peptide sequences compared to 271 in LMD-9 (Additional file 7 ), reflecting the roles of secreted proteins in different environmental niches of these closely related commensal versus dairy domesticated species.

Unlike PrtS and CpdB, the putative Mub does not have a SPaseI signal sequence at its $\mathrm{N}$-terminal end, but rather the YSIRK Gram-positive signal peptide [42] ([YF] SIRKxxxGxxS[VIA]; IPR005877), in this case YGIRKFSFGAASVAIA. There is little sequence homology to MucBP domain proteins from S. salivarius, however Mub shares $66 \%$ identity with a conserved hypothetical protein from L. fermentum that also contains a single MucBP binding domain and an LPXTG motif. The presence of this gene in LMD-9 may represent an ancestral remnant as Mubs are not encoded in the other $S$. thermophilus genomes. The loss of these functions in dairy bacteria has also been demonstrated in other lactic acid bacteria that have evolved to a specific ecological niche. For example, the small intestinal-associated L. acidophilus encodes for several Mubs compared to none in the closely related cheese starter strain of L. helveticus, demonstrating reductive evolution in their dairy environment [70]. Mubs in the case of streptococci may be important for pathogenesis of these strains, providing an advantage in regard to colonization. Loss of these virulence-like features is a common trait observed in S. thermophilus strains [9].

The third cell surface protein, $\mathrm{CpdB}$, is believed to convert non-transportable nucleotides produced by RNaseI to nucleosides which can then readily enter the cell and be utilized as a carbon source. $\mathrm{CpdB}$ has recently been reported as a virulence factor and was 
upregulated under iron starvation in S. suis [71]. Identification of this orthologous gene (SntA) in S. suis revealed a tripeptide RGD motif in CpdB [72]. This RGD motif has been demonstrated to bind to integrins of mammalian cells and hence may play a role in host interactions. Interestingly the CpdB of LMD-9 also contains an RGD motif starting at amino acid position 519. Both LMG 18311 and CNRZ1066 possessed a truncated CpdB that has an LPXTG motif but lacks an N-terminal signal peptide [16]. The CpdB of LMD-9 shows high homology (91\%) to the CpdB of S. salivarius and intermediate identity to orthologs from S. sanguinis (60\%) and S. suis (56\%). Compared to LMG 18311 and CNRZ1066, it is tempting to suggest that LMD-9 has as yet not undergone as extensive genomic decay in terms of genes that may play a beneficial role in the gastrointestinal tract such as mucin binding and utilization of metabolites, which has also been observed for probiotic lactobacilli [70]. This is in addition to an intact sortase in LMD-9 that allows processing of PrtS for cell surface exposure that potentially confer an advantage for growth of LMD-9 in milk.

\section{(v) Response to bacteriophage infection}

Several mechanisms exist within the $S$. thermophilus genome to combat bacteriophage infections. Bacteriophage resistance is usually conferred via non-specific point mutations in genes involving cell receptor sites [73]. In addition to other, more traditional bacteriophage resistance mechanisms, S. thermophilus LMD-9 possesses three separate CRISPR loci, and 14 cas genes [18]. The activity of CRISPR genes at the onset of phage DT1 exposure was determined by DNA microarray analysis for each of the 14 known CRISPR genes, spread among 3 separate loci in the $S$. thermophilus LMD-9 genome (Figure 1, circle 10). The phage response arrays indicated a transient increase in global gene expression, including that of CRISPR genes, 5 minutes post infection, followed by a decrease in gene expression (Additional file 9).

The increased expression of the CRISPR1 locus (specifically, cas 2 and cas 1 genes) is indicative of their higher activity during the phage response. CRISPR1 is known to have repeat degeneracy within the CRISPR gene sequence, with spacer size more highly conserved and in the highest number when compared to the other two loci [18]. This increase in spacer number and conservation in CRISPR1 is likely evidence of an effective mechanism to integrate novel spacers when faced with novel phage infection [74]. CRISPR3 plays a lesser role in phage response, and likewise less gene expression was observed. CRISPR2 has not been associated with active phage response $[18,74]$.

\section{(vi) Protocooperation with Lactobacillus bulgaricus}

During yogurt fermentation, protocooperation between S. thermophilus and L. bulgaricus results in rapid acidification of milk. As $S$. thermophilus is used in conjunction with $L$. bulgaricus for yogurt fermentations, the sequencing of both genomes revealed additional symbiotic or "protocooperative" relationship between both strains. The advantages conferred between both species have been discussed previously with the release of the L. bulgaricus genome sequence [52]. These include an extracellular cell wall bound proteinase (PrtB) encoded by L. bulgaricus and the biosynthetic pathways of folate, $p$-aminobenzoic acid and ornithine by $S$. thermophilus [52]. More recently, in silico analysis of both genomes predicted genes acquired by LGT for both species [38]. These LGT events included the transfer of an EPS biosynthesis cassette from S. thermophilus to L. bulgaricus, and genes for the metabolism of sulfur-containing amino acids from L. bulgaricus (or L. helveticus) to $S$. thermophilus.

In silico analysis of LMD-9 revealed the presence of a gene (STER0938) encoding a putative enzyme with conserved domain (COG0010) and a pfam domain (00491) related to the arginase family of enzymes. The putative arginase/agmatinase is likely involved in the biosynthesis of polyamine putrescine by the conversion of $\mathrm{L}$-arginine to L-ornithine and urea, and thus may contribute to protocooperation with L. bulgaricus through polyamine metabolism. This gene is uniquely present in S. thermophilus (Additional file 4) as no ortholog is found in other streptococci, L. lactis or lactobacilli. In fact, the next best sequence match in the current database was less than 30\% identity with orthologs from an archaea species isolated from metagenomic analysis of soil. Its low $\mathrm{G}+\mathrm{C}$ composition (28.2\%) confirms that the gene was horizontally acquired, presumably from another low GC microorganism.

The transcriptomics of $S$. thermophilus LMD-9 during milk fermentation in co-culture with $L$. bulgaricus revealed significant regulation of several amino acid metabolism and transport genes compared to the control (data not shown). This result was expected, as the presence of L. bulgaricus and its extracellular protease would increase the level of hydrolyzed proteins that would be available for use once the free amino acids from the milk media were depleted. Herve-Jimenez et al. [75] likewise showed that there was an upregulation of amino acid biosynthesis and nucleic acid metabolism in S. thermophilus when grown in co-culture with L. bulgaricus.

\section{Conclusions}

Although milk is a rich growth medium for many microorganisms, bacteria that grow and compete well in 
the milk environment must, at minimum, be able to use lactose as an energy source and milk protein as a source of amino acids. The adaptation of S. thermophilus LMD-9 to the milk environment is reflected by several observations from genomic and transcriptome analyses, including specialized systems for metabolizing lactose, the general absence of other carbohydrate metabolic systems, the presence of amino acid and peptide scavenging machinery, and numerous stress response and host defense mechanisms. Functional genome distribution analysis showed a close functional relationship between $S$. thermophilus and S. salivarius. Although the genome of $S$. thermophilus reflects a more simplified lifestyle compared to S. salivarius, both species shared several unique gene features (e.g. urease biogenesis, lactose metabolism) that are not universally present in other streptococci, which were possibly acquired by clonal populations from their natural ecosystems. Meanwhile, almost half of S. thermophilus LMD-9 unique genes have no orthologous counterpart among other streptococci. This indicates that LGT, the majority of which were likely mediated via transposable elements, served an important role in shaping the unique gene repertoire that potentially contribute to the fitness of LMD-9 in its specialized niches. The degree of sequence similarity of some of these alien genes, e.g. those involved in EPS biosynthesis and peptide transporters with microorganisms naturally found in the human intestinal environment suggests multiple transient habitats of LMD-9 beyond the restricted dairy environment. Compared to LMG 18311 and CNRZ1066, the notable presence of intact cell surface factors (mucus-binding protein, cyclonucleotide phosphodiesterase) in LMD-9 suggests that the latter strain has as yet not undergone as extensive genomic decay with regards to genes that may contribute to the interaction with human intestinal epithelium. This is in addition to unique EPS signature and functional bacteriocin production machinery that potentially expand the probiotic attributes of LMD-9.

\section{Additional material}

Additional file 1: Sequenced Streptococcus strains included in the Functional Genome Distribution (FGD) analysis

Additional file 2: Pseudogenes in the S. thermophilus LMD-9 genome

Additional file 3: S. thermophilus LMD-9 specific gene regions that are absent in both CNRZ1066 and LMG 18311 strains

Additional file 4: S. thermophilus-specific genes not present in

S. salivarius SK126 draft genome

Additional file 5: Unique genes among the sub-clusters of S. pneumoniae strains

Additional file 6: Unique genes among the sub-clusters of S. pyogenes strains
Additional file 7: ORFs with predicted signal peptide sequence in S. thermophilus LMD-9

Additional file 8: Two-component regulatory systems (2CRS) in S. thermophilus LMD-9

Additional file 9: CRISPR gene activity during phage infection

\section{Acknowledgment}

The genome sequencing of S. thermophilus LMD-9 was performed by the Joint Genome Institute, U.S. Department of Energy and by Fidelity Systems, Inc., as part of the Lactic Acid Bacteria Genome Consortium. We thank David Mills, Bart Wiemer, and Todd Klaenhammer for coordinating this effort. We are grateful to Todd Klaenhammer for providing resources to conduct carbohydrate utilization experiments. We also appreciate the technical assistance of Andrew Benson, Jaehyoung Kim, Zhijie Yang, and Joseph Nietfeldt at the University of Nebraska. YJG would like to thank Todd Klaenhammer and Evelyn Durmaz.

This article has been published as part of Microbial Cell Factories Volume 10 Supplement 1, 2011: Proceedings of the 10th Symposium on Lactic Acid Bacterium. The full contents of the supplement are available online at http:// www.microbialcellfactories.com/supplements/10/S1.

\section{Author details}

${ }^{1}$ Department of Food, Bioprocessing and Nutrition Sciences, North Carolina State University, Raleigh, North Carolina 27695, USA. ${ }^{2}$ School of Biological Sciences, University of Nebraska_Lincoln, Lincoln, Nebraska 68588, USA. ${ }^{3}$ AgResearch Limited, Grasslands Research Centre, Palmerston North, New Zealand. ${ }^{4}$ Riddet Institute, hosted by Massey University, Palmerston North, New Zealand. ${ }^{5}$ Department of Food Science and Technology, University of Nebraska-Lincoln, Lincoln, Nebraska 68583, USA.

\section{Authors' contributions}

YJG performed genome annotation, genome alignments, in silico and comparative genome analysis, and carbohydrate growth experiments. CG carried out microarray gene expression studies and transcriptome analysis. SOF performed in silico secretome and protocooperation analysis. EA conducted functional genome distribution analysis and comparative genome analysis. RH conceived the study, and participated in its design and coordination. All authors wrote, read and approved the final manuscript.

\section{Competing interests}

The authors declare that they have no competing interests.

Published: 30 August 2011

\section{References}

1. Orla-Jensen S: The lactic acid bacteria. Copenhagen: A. F. Host and Son; 1919.

2. Euzeby JP: List of Bacterial Names with Standing in Nomenclature: a folder available on the Internet. Int J Syst Bacteriol 1997, 47:590-592.

3. Ott A, Hugi A, Baumgartner M, Chaintreau A: Sensory investigation of yogurt flavor perception: mutual influence of volatiles and acidity. J Agric Food Chem 2000, 48:441-450.

4. de Vrese M, Stegelmann A, Richter B, Fenselau S, Laue C, Schrezenmeir J: Probiotics-compensation for lactase insufficiency. Am J Clin Nutr 2001, 73:421S-429S

5. Garcia-Albiach R, Jose M, de Felipe P, Angulo S, Morosini MI, Bravo D, Baquero F, del Campo R: Molecular analysis of yogurt containing Lactobacillus delbrueckii subsp. bulgaricus and Streptococcus thermophilus in human intestinal microbiota. Am J Clin Nutr 2008, 87:91-96.

6. Meyer AL, Elmadfa I, Herbacek I, Micksche M: Probiotic, as well as conventional yogurt, can enhance the stimulated production of proinflammatory cytokines. J Hum Nutr Diet 2007, 20:590-598.

7. Rodriguez C, Medici M, Rodriguez AV, Mozzi F, Font de Valdez G: Prevention of chronic gastritis by fermented milks made with exopolysaccharide-producing Streptococcus thermophilus strains. J Dairy Sci 2009, 92:2423-2434. 
8. Zhu Y, Xiao L, Shen D, Hao Y: Competition between yogurt probiotics and periodontal pathogens in vitro. Acta Odontol Scand 2010, 68:261-268.

9. Bolotin A, Quinquis B, Renault P, Sorokin A, Ehrlich SD, Kulakauskas S, Lapidus A, Goltsman E, Mazur M, Pusch GD, et al: Complete sequence and comparative genome analysis of the dairy bacterium Streptococcus thermophilus. Nat Biotechnol 2004, 22:1554-1558.

10. Makarova K, Slesarev A, Wolf Y, Sorokin A, Mirkin B, Koonin E, Pavlov A, Pavlova N, Karamychev V, Polouchine N, et al: Comparative genomics of the lactic acid bacteria. Proc Natl Acad Sci U S A 2006, 103:15611-15616.

11. Fontaine $L$, Boutry $C$, de Frahan $M H$, Delplace $B$, Fremaux $C$, Horvath $P$, Boyaval $\mathrm{P}$, Hols P: A novel pheromone quorum-sensing system controls the development of natural competence in Streptococcus thermophilus and Streptococcus salivarius. J Bacteriol 2010, 192:1444-1454.

12. Fontaine L, Boutry C, Guedon E, Guillot A, Ibrahim M, Grossiord B, Hols P: Quorum-sensing regulation of the production of Blp bacteriocins in Streptococcus thermophilus. J Bacteriol 2007, 189:7195-7205.

13. Fontaine $L$, Hols $P$ : The inhibitory spectrum of thermophilin 9 from Streptococcus thermophilus LMD-9 depends on the production of multiple peptides and the activity of $\mathrm{BlpG}(\mathrm{St})$, a thiol-disulfide oxidase. Appl Environ Microbiol 2008, 74:1102-1110.

14. Gardan R, Besset C, Guillot A, Gitton C, Monnet V: The oligopeptide transport system is essential for the development of natural competence in Streptococcus thermophilus strain LMD-9. J Bacteriol 2009, 191:4647-4655

15. Ibrahim M, Guillot A, Wessner F, Algaron F, Besset C, Courtin P, Gardan R, Monnet $V$ : Control of the transcription of a short gene encoding a cyclic peptide in Streptococcus thermophilus: a new quorum-sensing system? J Bacteriol 2007, 189:8844-8854.

16. Hols P, Hancy F, Fontaine L, Grossiord B, Prozzi D, Leblond-Bourget N, Decaris B, Bolotin A, Delorme C, Dusko Ehrlich S, et al: New insights in the molecular biology and physiology of Streptococcus thermophilus revealed by comparative genomics. FEMS Microbiol Rev 2005, 29:435-463.

17. Barrangou R, Fremaux $C$, Deveau H, Richards M, Boyaval P, Moineau S, Romero DA, Horvath P: CRISPR provides acquired resistance against viruses in prokaryotes. Science 2007, 315:1709-1712.

18. Horvath $\mathrm{P}$, Romero DA, Coute-Monvoisin AC, Richards M, Deveau H, Moineau S, Boyaval P, Fremaux C, Barrangou R: Diversity, activity, and evolution of CRISPR loci in Streptococcus thermophilus. J Bacteriol 2008, 190:1401-1412.

19. Sherman JM: The streptococci. Bacteriol Rev 1937, 1:3-97.

20. Rasmussen TB, Danielson M, Valina O, Johansen E, Pederson MB: The Streptococcus thermophilus core genome - a comparative genome hybridization study of 47 strains. Appl Environ Microbiol 2008, 74:4703-4710.

21. Altermann E, Klaenhammer TR: GAMOLA: a new local solution for sequence annotation and analyzing draft and finished prokaryotic genomes. Omics 2003, 7:161-169.

22. Rutherford K, Parkhill J, Crook J, Horsnell T, Rice P, Rajandream MA, Barrell B: Artemis: sequence visualization and annotation. Bioinformatics 2000 16:944-945.

23. Delcher AL, Bratke KA, Powers EC, Salzberg SL: Identifying bacterial genes and endosymbiont DNA with Glimmer. Bioinformatics 2007, 23:673-679.

24. Altschul SF, Madden TL, Schaffer AA, Zhang J, Zhang Z, Miller W, Lipman DJ: Gapped BLAST and PSI-BLAST: a new generation of protein database search programs. Nucleic Acids Res 1997, 25:3389-3402.

25. Tatusov RL, Fedorova ND, Jackson JD, Jacobs AR, Kiryutin B, Koonin EV, Krylov DM, Mazumder R, Mekhedov SL, Nikolskaya AN, et al: The COG database: an updated version includes eukaryotes. BMC Bioinformatics 2003, 4:41.

26. Eddy SR: A new generation of homology search tools based on probabilistic inference. Genome Inform 2009, 23:205-211.

27. Bateman A, Birney E, Cerruti L, Durbin R, Etwiller L, Eddy SR, GriffithsJones S, Howe KL, Marshall M, Sonnhammer EL: The Pfam protein families database. Nucleic Acids Res 2002, 30:276-280.

28. Krogh A, Larsson B, von Heijne G, Sonnhammer EL: Predicting transmembrane protein topology with a hidden Markov model: application to complete genomes. J Mol Biol 2001, 305:567-580.

29. Bendtsen JD, Nielsen H, von Heijne G, Brunak S: Improved prediction of signal peptides: SignalP 3.0. J Mol Biol 2004, 340:783-795.

30. Bendtsen JD, Nielsen H, Widdick D, Palmer T, Brunak S: Prediction of twinarginine signal peptides. BMC Bioinformatics 2005, 6:167.
31. Kerkhoven R, van Enckevort FH, Boekhorst J, Molenaar D, Siezen RJ: Visualization for genomics: the Microbial Genome Viewer. Bioinformatics 2004, 20:1812-1814.

32. Yang J, Wang J, Yao ZJ, Jin Q, Shen Y, Chen R: GenomeComp: a visualization tool for microbial genome comparison. J Microbiol Methods 2003, 54:423-426.

33. Altermann E, Klaenhammer TR: PathwayVoyager: pathway mapping using the Kyoto Encyclopedia of Genes and Genomes (KEGG) database. BMC Genomics 2005, 6:60.

34. Sneath PH, Sokal RR: Numerical taxonomy. Nature 1962, 193:855-860.

35. Tamura K, Dudley J, Nei M, Kumar S: MEGA4: Molecular Evolutionary Genetics Analysis (MEGA) software version 4.0. Mol Biol Evol 2007, 24:1596-1599.

36. Carver TJ, Rutherford KM, Berriman M, Rajandream MA, Barrell BG, Parkhill J: ACT: the Artemis Comparison Tool. Bioinformatics 2005, 21:3422-3423.

37. Monnet $C$, Ulve $V$, Sarthou AS, Irlinger F: Extraction of RNA from cheese without prior separation of microbial cells. Appl Environ Microbiol 2008, 74:5724-5730.

38. Liu M, Siezen RJ, Nauta A: In silico prediction of horizontal gene transfer events in Lactobacillus bulgaricus and Streptococcus thermophilus reveals protocooperation in yogurt manufacturing. Appl Environ Microbiol 2009, 75:4120-4129.

39. Delorme C, Bartholini C, Bolotine A, Ehrlich SD, Renault P: Emergence of a cell wall protease in the Streptococcus thermophilus population. Appl Environ Microbiol 2010, 76:451-460.

40. Fernandez A, Thibessard A, Borges F, Gintz B, Decaris B, Leblond-Bourget N: Characterization of oxidative stress-resistant mutants of Streptococcus thermophilus CNRZ368. Arch Microbiol 2004, 182:364-372.

41. Kawamura Y, Hou XG, Sultana F, Miura H, Ezaki T: Determination of $16 \mathrm{~S}$ rRNA sequences of Streptococcus mitis and Streptococcus gordonii and phylogenetic relationships among members of the genus Streptococcus. Int J Syst Bacteriol 1995, 45:406-408.

42. Tettelin H, Nelson KE, Paulsen IT, Eisen JA, Read TD, Peterson S, Heidelberg J, DeBoy RT, Haft DH, Dodson RJ, et al: Complete genome sequence of a virulent isolate of Streptococcus pneumoniae. Science 2001, 293:498-506.

43. Ding F, Tang P, Hsu MH, Cui P, Hu S, Yu J, Chiu CH: Genome evolution driven by host adaptations results in a more virulent and antimicrobialresistant Streptococcus pneumoniae serotype 14. BMC Genomics 2009, 10:158.

44. Smoot JC, Barbian KD, Van Gompel JJ, Smoot LM, Chaussee MS, Sylva GL, Sturdevant DE, Ricklefs SM, Porcella SF, Parkins LD, et al: Genome sequence and comparative microarray analysis of serotype M18 group A Streptococcus strains associated with acute rheumatic fever outbreaks. Proc Natl Acad Sci U S A 2002, 99:4668-4673.

45. Nakagawa I, Kurokawa K, Yamashita A, Nakata M, Tomiyasu Y, Okahashi N, Kawabata S, Yamazaki K, Shiba T, Yasunaga T, et al: Genome sequence of an M3 strain of Streptococcus pyogenes reveals a large-scale genomic rearrangement in invasive strains and new insights into phage evolution. Genome Res 2003, 13:1042-1055.

46. Beres SB, Sylva GL, Sturdevant DE, Granville CN, Liu M, Ricklefs SM, Whitney AR, Parkins LD, Hoe NP, Adams GJ, et al: Genome-wide molecular dissection of serotype M3 group A Streptococcus strains causing two epidemics of invasive infections. Proc Natl Acad Sci U S A 2004, 101:11833-11838.

47. Holden MT, Scott A, Cherevach I, Chillingworth T, Churcher C, Cronin A, Dowd L, Feltwell T, Hamlin N, Holroyd S, et al: Complete genome of acute rheumatic fever-associated serotype M5 Streptococcus pyogenes strain Manfredo. J Bacterio/ 2007, 189:1473-1477.

48. McShan WM, Ferretti JJ, Karasawa T, Suvorov AN, Lin S, Qin B, Jia H, Kenton S, Najar F, Wu H, et al: Genome sequence of a nephritogenic and highly transformable M49 strain of Streptococcus pyogenes. J Bacteriol 2008, 190:7773-7785

49. Arioli S, Monnet C, Guglielmetti S, Parini C, De Noni I, Hogenboom J, Halami PM, Mora D: Aspartate biosynthesis is essential for the growth of Streptococcus thermophilus in milk, and aspartate availability modulates the level of urease activity. App/ Environ Microbiol 2007, 73:5789-5796.

50. Ajdic D, McShan WM, McLaughlin RE, Savic G, Chang J, Carson MB, Primeaux C, Tian R, Kenton S, Jia H, et al: Genome sequence of Streptococcus mutans UA159, a cariogenic dental pathogen. Proc Natl Acad Sci U S A 2002, 99:14434-14439. 
51. Xu P, Alves JM, Kitten T, Brown A, Chen Z, Ozaki LS, Manque P, Ge X, Serrano MG, Puiu D, et al: Genome of the opportunistic pathogen Streptococcus sanguinis. J Bacteriol 2007, 189:3166-3175.

52. van de Guchte M, Penaud S, Grimaldi C, Barbe V, Bryson K, Nicolas P, Robert C, Oztas S, Mangenot S, Couloux A, et al: The complete genome sequence of Lactobacillus bulgaricus reveals extensive and ongoing reductive evolution. Proc Natl Acad Sci U S A 2006, 103:9274-9279.

53. Kuhn A: From the Sec complex to the membrane insertase YidC. Biol Chem 2009, 390:701-706.

54. Abe A: [Bacterial secretion systems: their function and contribution to disease process]. Kansenshogaku Zasshi 2009, 83:94-100.

55. Bourgoin F, Pluvinet A, Gintz B, Decaris B, Guedon G: Are horizontal transfers involved in the evolution of the Streptococcus thermophilus exopolysaccharide synthesis loci? Gene 1999, 233:151-161.

56. Broadbent JR, McMahon DJ, Welker DL, Oberg CJ, Moineau S: Biochemistry, genetics, and applications of exopolysaccharide production in Streptococcus thermophilus: a review. J Dairy Sci 2003, 86:407-423.

57. Makino S, Ikegami S, Kano H, Sashihara T, Sugano H, Horiuchi H, Saito T, Oda M: Immunomodulatory effects of polysaccharides produced by Lactobacillus delbrueckii ssp. bulgaricus OLL1073R-1. J Dairy Sci 2006, 89:2873-2881.

58. van den Bogaard PT, Hols P, Kuipers OP, Kleerebezem M, de Vos WM: Sugar utilisation and conservation of the gal-lac gene cluster in Streptococcus thermophilus. Syst Appl Microbiol 2004, 27:10-17.

59. Vaillancourt K, Moineau S, Frenette M, Lessard C, Vadeboncoeur C: Galactose and lactose genes from the galactose-positive bacterium Streptococcus salivarius and the phylogenetically related galactosenegative bacterium Streptococcus thermophilus: organization, sequence, transcription, and activity of the gal gene products. J Bacteriol 2002, 184:785-793.

60. Brooker JD, McCarthy JM: Gene knockout of the intracellular amylase gene by homologous recombination in Streptococcus bovis. Curr Microbiol 1997, 35:133-138.

61. Satoh E, Uchimura T, Kudo T, Komagata K: Purification, characterization, and nucleotide sequence of an intracellular maltotriose-producing alpha-amylase from Streptococcus bovis 148. Appl Environ Microbiol 1997, 63:4941-4944.

62. Simpson CL, Russell RR: Intracellular alpha-amylase of Streptococcus mutans. J Bacterio/ 1998, 180:4711-4717.

63. Bonifait L, de la Cruz Dominguez-Punaro M, Vaillancourt K, Bart C, Slater J, Frenette M, Gottschalk M, Grenier D: The cell envelope subtilisin-like proteinase is a virulence determinant for Streptococcus suis. BMC Microbiol 10:42.

64. Courtin P, Monnet V, Rul F: Cell-wall proteinases PrtS and PrtB have a different role in Streptococcus thermophilus/Lactobacillus bulgaricus mixed cultures in milk. Microbiology 2002, 148:3413-3421.

65. Geis A, El Demerdash HA, Heller KJ: Sequence analysis and characterization of plasmids from Streptococcus thermophilus. Plasmid 2003, 50:53-69.

66. Solow BT, Somkuti GA: Comparison of low-molecular-weight heat stress proteins encoded on plasmids in different strains of Streptococcus thermophilus. Curr Microbiol 2000, 41:177-181.

67. Wouters JA, Sanders JW, Kok J, de Vos WM, Kuipers OP, Abee T: Clustered organization and transcriptional analysis of a family of five csp genes of Lactococcus lactis MG1363. Microbiology 1998, 144:2885-2893.

68. Yamaguchi M, Terao Y, Ogawa T, Takahashi T, Hamada S, Kawabata S: Role of Streptococcus sanguinis sortase A in bacterial colonization. Microbes Infect 2006, 8:2791-2796.

69. Leigh JA, Egan SA, Ward PN, Field TR, Coffey TJ: Sortase anchored proteins of Streptococcus uberis play major roles in the pathogenesis of bovine mastitis in dairy cattle. Vet Res 2010, 41:63.

70. Ventura M, O'Flaherty S, Claesson MJ, Turroni F, Klaenhammer TR, van Sinderen D, OToole PW: Genome-scale analyses of health-promoting bacteria: probiogenomics. Nat Rev Microbiol 2009, 7:61-71.

71. Li W, Liu L, Chen H, Zhou R: Identification of Streptococcus suis genes preferentially expressed under iron starvation by selective capture of transcribed sequences. FEMS Microbiol Lett 2009, 292:123-133.

72. Osaki M, Takamatsu D, Shimoji Y, Sekizaki T: Characterization of Streptococcus suis genes encoding proteins homologous to sortase of gram-positive bacteria. J Bacteriol 2002, 184:971-982.
73. Pederson MB, Iverson SL, Sorenson Kl, Johansen E: The long and winding road from the research laboratory to industrial applications of lactic acid bacteria. FEMS Microbiol Rev 2005, 29:611-624.

74. Mills S, Griffin C, Coffey A, Mijer WC, Hafkamp B, Ross RP: CRISPR analysis of bacteriphage-insensitive mutants (BIMs) of industrial Streptococcus thermophilus - implications for starter design. J Appl Microbiol 2010, 108:945-955

75. Herve-Jimenez L, Guillouard I, Guedon E, Boudebbouze S, Hols P, Monnet V, Maguin E, Rul F: Postgenomic analysis of Streptococcus thermophilus cocultivated in milk with Lactobacillus delbrueckii subsp. bulgaricus: involvement of nitrogen, purine, and iron metabolism. Appl Environ Microbiol 2009, 75:2062-2073.

doi:10.1186/1475-2859-10-S1-S22

Cite this article as: Goh et al:: Specialized adaptation of a lactic acid bacterium to the milk environment: the comparative genomics of Streptococcus thermophilus LMD-9. Microbial Cell Factories 2011 10(Suppl 1):S22.

\section{Submit your next manuscript to BioMed Central and take full advantage of:}

- Convenient online submission

- Thorough peer review

- No space constraints or color figure charges

- Immediate publication on acceptance

- Inclusion in PubMed, CAS, Scopus and Google Scholar

- Research which is freely available for redistribution

Submit your manuscript at www.biomedcentral.com/submit
C Biomed Central 